\title{
«Nell'ombra delle mie ali d'uomo»: echi autobiografici, letterari e giornalistici della partecipazione di Gabriele d'Annunzio alla Prima guerra mondiale
}

\author{
Antonio ZOLLINO \\ Università Cattolica di Milano $^{1}$ \\ antonio.zollino@unicatt.it
}

Recibido: 23/04/2015

Aceptado: 03/06/2015

\section{RIASSUNTO}

La figura e l'opera di Gabriele d'Annunzio riveste, come è noto, un ruolo cruciale nella partecipazione dell'Italia al primo conflitto mondiale; altrettanto note sono le gesta del poeta-soldato durante le azioni di guerra, così come la produzione oratoria e poetica dedicata con particolare intensità, negli stessi anni, alla propaganda bellica. L'articolo si propone di rendere conto dell'esperienza bellica dannunziana filtrata attraverso alcuni riflessi autobiografici e così come si presenta nell'opinione di alcuni importanti intellettuali italiani.

Parole chiave: d'Annunzio soldato, Ettore Cozzani, prima guerra mondiale, discorso di Quarto, beffa di Buccari.

«Nell'ombra delle mie ali d'uomo» (In the shadow of my human wings): Autobiographical, literary and journalistic echos of the Gabriele d'Annunzio's participation in the First World War

\begin{abstract}
As it is well known, Gabriele d'Annunzio, his work and his figure play a very relevant role in determining the Italian participation in the First World War. The exploits of the poetsoldier at the war maneuvers and his poetic and oratory production, which, in those years, presented a strong propagandistic effort in favour of the war, are very well known. The aim of this article is to analyze the autobiographical reconstruction of the d'Annunzio's war experience and its evaluation in the thought of some relevant Italian intellectuals.
\end{abstract} Milano.

${ }^{1}$ Dipartimento di Italianistica e comparatistica, Largo Agostino Gemelli 1, I-20123 - 
Key words: d'Annunzio as soldier, Ettore Cozzani, First World War, discourse at Quarto, joke of Buccari.

SOMMARIO: 1. Il discorso di Quarto 2. Il poeta soldato nel mirino degli scrittori.

\section{IL DISCORSO DI QUARTO}

Il 5 maggio 1915, inaugurando il monumento garibaldino dello scultore Eugenio Baroni, Gabriele d'Annunzio commemora a Genova l'anniversario della partenza della spedizione dei Mille dallo scoglio di Quarto: si tratta di un evento molto sentito dall'intera nazione, perché il momento presente finisce per collimare con non pochi aspetti di quello storico, per finalità, simbologie e rituali patriottici. Il poeta oltretutto è accompagnato da Peppino Garibaldi, figlio dell'Eroe dei due mondi, emblema vivente della continuità degli ideali risorgimentali, che verrà a Genova con i suoi legionari reduci dall'eroica - e se vogliamo anche abbastanza dissennata - resistenza nelle Argonne. Per quanto riguarda d'Annunzio, invece, si concretizza un clamoroso ritorno in Italia, dopo la fuga improvvisa di cinque anni prima, assai meno gloriosa, dovuta com'era all'incalzare di creditori poco inclini a contraccambiare la stabilità dei propri bilanci economici con le ragioni della poesia e del vivere d'arte dannunziano. Il rientro in patria in occasione dell'evento è stato organizzato da un vivace letterato della Spezia, il direttore dell'Eroica Ettore Cozzani. Molti se ne attribuiranno in seguito la paternità ${ }^{2}$, approfittando del contegno piuttosto schivo del letterato spezzino, tanto modesto da rievocare in un suo volume la preparazione dell'evento senza accennare esplicitamente a se stesso:

Un giorno della primavera del 1915, nel suo studio remoto di Rue de Geoffroy, a Parigi, con Peppino Garibaldi, preso come in un'atmosfera di leggenda, egli si artiglia il cervello per trovare un modo di ritornare in Italia che valga un ammonimento, e che tagli il nodo della sorte, quand'ecco gli giunge un messaggio: un giovane poeta gli annuncia dall'Italia che, nel prossimo anniversario della partenza dei Mille da Quarto, si scoprirà sullo scoglio un grande bronzo, a cui gli eventi danno un significato, per la folla improvviso, ma a lungo meditato e preparato nel cuore dello scultore: è la risurrezione dei morti: gli eroi di tutte le cospirazioni e le guerre dell'indipendenza ribalzano dalla terra, stracciando i loro sudarii; e Garibaldi è fra loro: li raccoglie, li regge e li guida, simili a un flutto che urti la sua persona come uno scoglio. La vittoria giovane, perché garibaldina, scaturisce dal gruppo affannoso, e incorona il Condottiere.

Si vuole che il poeta torni in patria, sbarcando sul lido «fatale», e interpreti il bronzo e compia il prodigio, davvero: la risurrezione dei morti s'inizi per comandamento di lui. (Cozzani 1930: 34-35)

${ }^{2}$ Fra cui il ministro e letterato Ferdinando Martini, peraltro spesso assai critico nei confronti delle iniziative dannunziane (Antongini 1938: 677-8). 
È tuttavia lo stesso d'Annunzio a fornire l'identità del «giovane poeta», ovvero la prova della fondamentale mediazione di Cozzani (che fra l'altro, di lì a poco diverrà l'editore della dannunziana Crociata degi innocenti ${ }^{3}$ ), in una pagina dei propri Taccuini:

\section{Domenica 7 marzo}

Stamani mi levo per tempo. Non ho più febbre ma sono ancora soffrente, infastidito dal piccolo male umiliante. Peppino Garibaldi deve venire alle dieci.

Cerco le fotografie del monumento di Eugenio Baroni ai Mille, inviatemi da Ettore Cozzani. È un monumento marino, modellato dal flutto decumano. Gli eroi risorgono con un ritmo di marea $[\ldots]$

Mi ricordo di non aver ancora aperto la lettera che le accompagna. La cerco. L'apro, la leggo. E tutto, ecco, si rischiara!

V'è certo una provvidenza apollinea. Quel che mi è offerto, è tal cosa che risolve tutti i dubbii e tutte le perplessità, ci salva da ogni errore, da ogni deformazione, dal pericolo dei contrattempi, dei dissensi, dei moti intempestivi.

Il municipio di Genova mi chiede di parlare al popolo d'Italia il 5 maggio, all'inaugurazione del Monumento, nel giorno anniversario della dipartita meravigliosa.

Quale più grande occasione?

Andrò, condurrò meco la legione garibaldina, il flutto rosso. I fati saranno maturi. Nessuna opposizione, nessuna sorpresa saranno più da temere. La radunanza non sarà soltanto consentita ma favorita. Da ogni parte d'Italia tutti gli spiriti rossi potranno accorrere. Una forza imperiosa si accalcherà intorno al bronzo perenne. Dove i Mille salparono, quivi i nuovi Mille approderanno. Il movimento sarà irresistibile. Dallo scoglio di Quarto l'esercito d'Italia muoverà verso i confini [...]

Mi sembra d'esser ripreso dalla febbre. La fronte mi arde.

Ecco alfine la ricompensa a questa mia attesa troppo paziente e tanto malinconica, a questo troppo lungo esilio.

Una visione di poesia può tradursi in realtà militante! (d'Annunzio 1965: 714-715)

Per quanto piuttosto enfatiche, le previsioni di d'Annunzio si realizzeranno con impressionante puntualità, tanto che l'evento viene ancora oggi ricordato da alcuni, certo in modo semplicistico ed esagerato, come determinante nel favorire l'ingresso in guerra dell'Italia. Di fatto, premeranno per il coinvolgimento italiano nella guerra in corso ampi settori della società italiana del tempo, dagli imprenditori legati all'industria navale e metalmeccanica all'università, con allievi e docenti spesso in prima fila ${ }^{4}$ : all'evento di Quarto parteciparono infatti circa quattromila studenti, e anche nel resto d'Italia gli studenti non mancarono di partecipare

${ }^{3}$ Pubblicata ne L'Eroica, VI e VII, agosto e settembre 1915.

${ }^{4}$ A testimonianza dell'impegno professorale nel sostenere le ragioni della guerra, si veda il volume dell'Associazione nazionale fra i professori universitari, La nostra guerra, con scritti di G. Del Vecchio, P. Fedozzi, C. Errera, P. S. Leich, L. Bianchi, P. Bonfante, G. Arias, A. Solmi, G. Albini (Firenze, Tipografia domenicana 1915). 
appassionatamente a parallele manifestazioni interventiste. Particolarmente spassosa, in proposito, la rassegna dell'episodio ne La meccanica (1928-29) di Carlo Emilio Gadda:

il poeta della canzone al re giovine rivenne di terra di Francia, araldo delle fulgide armi; e toccò ai Genovesi l'onor primo d'averlo lor ospite, leggermente allungatasi la faccia loro quando all'esimio assessore alle finanze gli presentarono tutto il codazzo sansebastianesco e poi il conto del Monopole-Heidsieck [sic].

Gli studenti a Milano ed altrove, avevano gridato 'Morte a Giolitti', 'Viva la guerra!', 'Viva d'Annunzio', e noi stessi non lesinammo la voce de' polmonacci nostri di allora, in sì fortunata concomitanza d'eventi. (Gadda 1974: 88)

Difficile, a giudicare dal racconto dello stesso Cozzani (1963) e da altri resoconti, dare oggi un'idea dell'intensa giornata genovese, trascorsa nel segno di un entusiasmo e di un coinvolgimento di larghi settori della società civile difficilmente riscontrabili ai giorni nostri. Il discorso dannunziano fu largamente sommerso dalle acclamazioni e dai continui interventi delle sirene delle navi antistanti: è lecito supporre, pertanto, che i significati, peraltro complicati dalle usuali perifrasi e dai numerosi riferimenti eruditi, siano difficilmente pervenuti alla piena comprensione degli astanti. Nondimeno, si tratta di un discorso interessante, in cui l'ekfrasis del gruppo scultoreo si presta ad avallare il concetto della resurrezione degli eroi: il passato non esiste, il tempo è abolito in quanto l'impresa iniziata dai Mille attende ancora di essere completata, Garibaldi è visto, in chiave chiaramente cristologica, come il Salvatore: «Uomo egli fu, uomo tra uomini. E voi lo vedeste, santissimi vecchi, lo vedeste da presso come la Veronica vide il Cristo in passione. Il suo volto vero è impresso nella vostra anima come nel sudario il volto del Salvatore. Nessuna ombra l'offusca» (d'Annunzio 1947: 16).

La conclusione del discorso dannunziano è in effetti affidata a un'esibita riscrittura dell'evangelico Discorso della montagna tanto più irriverente se pensiamo che in quest'ultimo erano «beati gli operatori di pace». Per d'Annunzio invece la riscrittura ha fini del tutto laici, proponendosi di chiamare a raccolta tutte le forze della società italiana affinché si ritrovino concordi nell'intento bellico. Nel fare ciò, d'Annunzio non manca di porsi in un ottica di palese rovesciamento rispetto alla celebre pagina del Vangelo: sono, fra gli altri, «beati quelli che più hanno, perché più potranno dare, più potranno ardere» e altresì «Beati quelli che, avendo ieri gridato contro l'evento, accetteranno in silenzio l'alta necessità e non più vorranno essere gli ultimi ma i primi». Nella strategia oratoria dannunziana, il rovesciamento avviene comunque all'interno di una struttura liturgica che garantisce la trasmissione dei contenuti presso un ampio pubblico ed è appena il caso di notare come d'Annunzio abbia da tempo esperito con innegabile successo, nella propria opera letteraria, la commistione di eros e religione: ora viene meno l'elemento erotico e vi subentra quello eroico, assecondando un procedimento già attivo nelle Canzoni delle gesta d'Oltremare, pubblicate nel 1912 per celebrare la conquista coloniale libica. Sarà peraltro caratteristica costante dell'azione politica dannunziana quella di offrire un retroterra religioso ai propri progetti: così ad 
esempio avverrà per la frequente evocazione di San Francesco sia in ambito bellico che a sostegno delle teorie espansionistiche sulla sponda adriatica orientale (Mariano 1978).

Innumerevoli sono i riflessi giornalistici, anche al di là dei confini italiani ${ }^{5}$, del discorso dannunziano; per produrre un unico esempio, Ugo Ojetti descrive così, sulla prima pagina del Corriere della sera del 6 maggio, la discesa dal palco dell'oratore:

Fra gli evviva, gli abbracci, lo sventolìo delle bandiere, l'agitar dei cappelli, Gabriele d'Annunzio scende a stento la gradinata. Onde di folla lo spingono verso il palco reale, dove bisogna sollevarlo a braccia perché ogni altro varco per porlo in salvo è chiuso. Subito sull'alto del monumento viene posata una grande targa rossa tra due palme, e sulla targa è scritto a grandi lettere: Trieste. (ora in Ojetti 1957: 88)

\section{IL POETA SOLDATO NEL MIRINO DEGLI SCRITTORI}

Non mancavano però, com'è noto, le opinioni di segno opposto: così, nel 1916 Alfredo Panzini aveva fornito un chiaro riflesso della diffidenza nei confronti dell'impegno bellicista del Poeta in un interessante romanzo oggi del tutto dimenticato, La Madonna di Mamà. Ne è protagonista un giovane precettore catapultato dalla patria rustica al salotto cittadino della marchesa Barberina (situazione che prefigura quella dispiegata, come vedremo fra breve, nel Trono dei poveri di Marino Moretti). Fra gli abituali frequentatori del salotto prevalgono «intellettualità esotiche» e nelle conversazioni, più di quelli di Dante e Leonardo da Vinci, «ricorreva il nome di Gabriele d'Annunzio, di cui sapevano più cose che non ne sapesse forse quel poeta medesimo» (Panzini 1916: 111). Nell'imminenza della guerra, tuttavia, d'Annunzio viene evocato in questo modo nel dialogo fra Barberina e un cosiddetto «commendatore $\mathrm{X}^{* * *}$ », «uomo politico»:

- E cosa salta in mente adesso al d'Annunzio di venire in Italia? Stava così bene in Francia...

Qui il commendatore non sapeva che rispondere, se non che i poeti sono disordinati loro, e mettono il disordine da per tutto.

(Quel poeta era, infatti, venuto di Francia in Italia a destare il gran fantasma di Roma.)

- Ma lasciatela stare Roma, che appartiene alla storia antica - diceva donna Barberina.

- La guerra dichiarata per un menestrello! Enorme, inaudito! - esclamava il commendatore, agitando le palme in alto, qua e là dalle orecchie. (Panzini 1916: 281)

Panzini è assai tiepido rispetto all'entrata in guerra dell'Italia né si può certo annoverare tra i fanatici ammiratori del Poeta, eppure nel suo Diario sentimentale, dedicato alla memoria di Renato Serra, non sembra del tutto immune dalla

\footnotetext{
${ }^{5} \mathrm{Si}$ veda ad esempio, per la risonanza sulla stampa francese, Ostenc (1989: 91-93).
} 
fascinazione di quest'ultimo, anche se l'ironia lo trattiene da un'adesione poco meditata. Così nella pagina del 18 maggio 1915, a proposito dell'orazione che il Poeta ha tenuto il giorno prima Dalla ringhiera del Campidoglio (d'Annunzio 1947: 53-60), annota:

Discorso del d'Annunzio ai Romani. Pare San Paolo. La crosta letteraria è caduta. L'uomo è trasfigurato! È il politico, il deputato d'Italia per conclamazione. Felice te, o Poeta, che hai la forza di non più considerare per una cosa sola considerare!

Allora l'uomo non è finito, - come dicono i letterati - sed resurrexit! Un uomo, vissuto sino oltre i cinquant'anni in compagnia delle debilitanti Muse, e che conserva i nervi a posto, tesi all'azione, è un gran fenomeno!

Ah, sii il navarca, lo stratega, il despota!

Io credo che egli sia sincero come pochi altri; ma egli è tradito da non so che cosa che ha dentro, per cui come a Mida in oro, in lui tutto si trasmuta in sensazione di bellezza!

Questa Italia in armi è bella!

Mi pare che il genio del d'Annunzio sia così grande e proteiforme che egli stesso è dominato dal suo genio.

Dicono i maligni che gli hanno pagato i débiti; ma è pur sempre un gran fenomeno! Eppoi? Macché debiti! Un poeta non ha debiti, e quando dice ai creditori: «non mi seccate», non deve essere seccato.

$\mathrm{Ma}$, oh ironia, maligna dea! Mi si disegnano in mente, a proposito dei discorsi [di] d'Annunzio, le parole dell'uomo d'affari col quale conversai ieri:

«Volete chiamare molto popolo nel baraccone? Esponete manifesti con teste di leoni, grandi inscì. Denter ghe di gatt! II popolo paga, ride ed è contento». (Panzini 1923: 216217)

Lo stesso Renato Serra nell'Esame di coscienza di un letterato, in pagine scritte fra il 20 e il 25 marzo 1915, si era soffermato sulla figura di d'Annunzio all'interno della generale mobilitazione intellettuale in occasione dell'evento bellico; il suo caso conferma la tesi generale dell'Esame, secondo cui «è inutile aspettare delle trasformazioni o dei rinnovamenti dalla guerra» (Serra 1973: 40-41).

Così da noi: d'Annunzio, per esempio, a cui pensiamo con un certo orgoglio e quasi con simpatia da quando quella sua molto privata e curiosa «cattività in Babilonia» è diventata nel corso degli avvenimenti una espressione simbolica dell'Italia esiliata col cuore sui campi dove si difende un'altra volta la civiltà latina; e il suo ritorno ha un significato, che ci fa sperare e dubitar tutti quanti. Certo d'Annunzio ha guadagnato in questo momento: ha ripreso fra noi: è ritornato al posto, da cui pareva scaduto. In realtà, con tutto il favore delle circostanze e della fortuna, non è poi cresciuto di nulla: non ha fatto niente che sia degno di quell'apparente ingrandimento morale: per una lettera, da Parigi assediata, ricca e rotta magnificamente di colore, quante odi su la resurrezione latina, e frasi e parole odiosamente vecchie e false; come se niente potesse essere cambiato mai per lui! (Serra 1973: 40-41)

Pur non considerando la grande novità di alcune opere del periodo francese, dalla Contemplazione della morte alla Leda senza cigno, Serra denuncia chiaramente nel d'Annunzio bellico l'uso retorico di una fraseologia consunta, tale 
da offuscare l'ipotesi di suo «apparente ingrandimento morale». Ci fu tuttavia chi rimase ancor meno convinto dal verboso e bellicoso ritorno di d'Annunzio, come Giuseppe De Robertis fa chiaramente capire nell'articolo vociano del 15 agosto 1915 «d'Annunzio ha parlato», di cui conviene qui riportare un ampio stralcio:

Quest'accidente di Gabriele s'è sfogato dopo l'esilio. Una volta rimesso il piede in Italia, ci ha preso gusto. Ha parlato, ha parlato, da stordire, come una cicala! In tutti i luoghi. Sul mare, nei musei, per le vie, dai balconi, nei giardini, nell'andare e nell'uscire dal parlamento. Una pazzia, vi dico.

Oggi, grazie a Dio, si riposa un poco; ma chi sa mai quali discorsi prepara per dopo la guerra; e come canterà allora. Io non mi ci vorrei trovare. La patria va bene; ma d'Annunzio no. Questo civettone che pare abbia cominciato a posta dalle Odi navali la sua carriera di poeta civile per aver diritto oggi primo alla parola, dacché la morte l'ha sbarazzato di Giosuè; e anche del suo fratello minore e maggiore, che un po' gli avrebbe dato noia, in quest'occasione.

Ora, santissimo Iddio; quest'Italia di d'Annunzio; magari non così grande come proprio lui dice; ma più modesta; più piccolina; un'Italia per la sua statura insomma; io, con tutto il gran sfolgorio di sole non la vedo. Potrei dire, non l'ho mai veduta [...].

Sapevamo che d'Annunzio aspettava di venire in Italia, quasi in trionfo.

Mancò alla prima giornata quella solennità che forse s'augurava, al suo orgoglio; con il $\mathrm{Re}$, Salandra, e tutto il Senato centenario.

Ma si rifece dell'umiliazione.

Parlò invece di una dieci volte.

Era certo che la cosa avrebbe raggiunto il suo effetto.

Ora può dichiararsi soddisfatto.

L'Italia lo ha acclamato poeta della sua più grande guerra.

E come parata e messa in iscena solenne, vada.

Anche per un resultato materiale e misurabile d'entusiasmo che dicono giovi per l'occasione.

Diamo dunque alla cosa un valore provvisorio

$[\ldots]$

Ma, fuori di queste considerazioni economiche, buone da apprezzare l'eloquenza di un avvocato di difesa, vediamo di trovare, se possibile, appiglio a un discorso e a ragioni meno superficiali.

d'Annunzio non ha detto una sola parola necessaria.

$[\ldots]$

d'Annunzio ha falsificato la realtà.

Gli bastava parlare in nome della sua stessa natura e ferinità animale, che è poi il principio della nostra razza giovine, per aprire uno sbocco a espressioni di eloquenza non intempestiva.

Tutti, in parte, si sarebbero riconosciuti in lui.

Per questo piacere fisico della guerra.

Il cacciatore sardo e l'aspro lombardo scavalcatore di monti.

Invece è voluto tornare ai Greci e ai Romani. E avesse almeno inteso di documentare per via di tradizione il nostro diritto all'intelligenza, per cui siamo insorti.

Ha ricordato i soliti nomi, e Garibaldi e i garibaldini da cui ci sentiamo tanto lontani, con questa guerra matematica.

Ha gridato eroi gli uomini più comuni. 
Ha giocato d'impostura in nome dell'ora presente e dell'Italia.

Ha rinnegato la parte migliore di sé.

S'è presentato al cospetto della patria e del mare con la sua carta scritta; e ha lasciato a casa l'anima.

Non guardiamo all'improvviso entusiasmo, già di per sé così pronto a scoppiare.

Pensiamo che ci poteva essere concessa un'ora di grandezza, e l'abbiamo persa per sempre. (ora in Scalia 1973: 555-560)

L'acredine del critico, che forse avrebbe avuto un senso se dispiegata in un discorso avverso alla guerra (mentre De Robertis è favorevole al conflitto), sembra tuttavia non mordere più di tanto e finisce anzi per offrire una nuova cassa di risonanza alla smania di presenzialismo non senza motivo presunta nel bersaglio polemico (Zollino 2014: 82-83). Di fatto, un volta apertosi anche il fronte italiano nella grande guerra, d'Annunzio potrà disporre di un ennesimo palcoscenico su cui esibire la propria ventura: questa volta, però, sarà difficile distinguere le trovate dell'attore dall'effettiva pericolosità dello scena e dello spettacolo. Nonostante ciò, molti, agitati da personale e comprensibile fastidio nei confronti della pervadenza del personaggio dannunziano, continueranno a sottolineare proprio l'istrionismo e la verbosità del «Vate grammofono», «massima cloaca di tutto il letteratume» (Campana 1985: 233), per usare un paio di colorite espressioni di Dino Campana, sul cui larvato dannunzianesimo sussistono peraltro ben pochi dubbi. Ma c'è anche chi, come il moralista salernitano Giovanni Lanzalone, sa distaccare l'esperienza del poeta da quella del patriota e, con i versi dedicati A Gabriele d'Annunzio, auspica che l'opera risulti in qualche modo purificata dal fuoco della guerra:

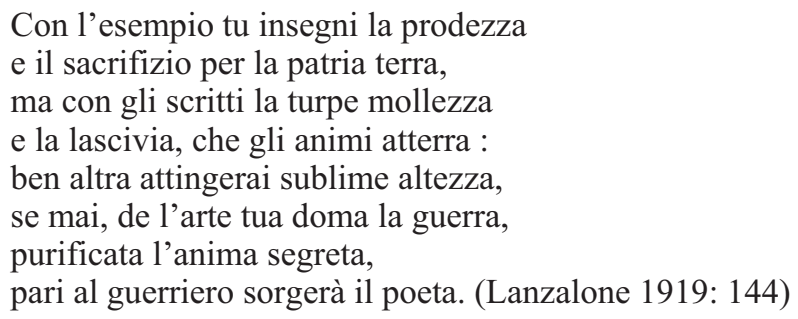

Coerentemente con i propri proclami, d'Annunzio, dopo il fatidico 24 maggio, aveva richiesto di essere arruolato come volontario alla bella età di 53 anni: la risposta ministeriale arrivò il 19 giugno 1915 con un bollettino che lo assegnava quale tenente di complemento nei Lancieri di Novara al quartier generale del duca di Aosta; ma già a luglio lo ritroviamo a Venezia come marinaio volontario e presto Gabriele manifesta l'intenzione di essere impiegato anche in operazioni aereonautiche. L'intento governativo è quello di preservarlo dalle azioni dirette per utilizzarne la figura e le doti oratorie a fini propagandistici. E tuttavia d'Annunzio si ribella a un simile ruolo che avverte come marginale: il 30 luglio scrive al presidente del consiglio dei ministri, secondo l'espressione di Angelo Sodini, una 
«fierissima lettera di dodici grandi fogli» (Sodini 1931: 433) in cui chiedeva con toni accorati di essere finalmente impiegato a tutti gli effetti:

Ella sa con quanta impazienza io abbia chiesto l'onore di servire la mia Patria in altri campi. Ella sa che tutta la mia vita io ho aspettato quest'ora. Avendo vissuto con tristezza e con ira tra un popolo incurante di gloria, ecco che finalmente assisto a un miracolo il quale risponde alla mia implacabile aspettazione. La gloria è ridivenuta il cielo stesso d'Italia. L'ora dei grandi fatti è suonata per quel popolo, l'ora del mio sangue è venuta per me. Fino ad oggi, se bene il mio novo canto sia atteso da molti, io non ho potuto comporre un poema sì vero. Ho l'orrore del lavoro immobile: della penna, dell'inchiostro, della carta, di tutte queste cose divenute oggi vane. La febbre dell'azione mi divora. Il pericolo è il solo dio lampeggiante a cui mi piace di consacrare la mia poesia inespressa ... Per trent'anni io ho gridato "armi armi armi", io ho gridato "navi navi navi". I soldati e i marinai se lo ricordano, lo sanno. Essi mi considerano come un fratello che li esprima ... L'Ammiraglio Cutinelli crede che da ora in poi mi sia vietato di partecipare a imprese pericolose. Non so dirLe quanto io ne sia addolorato, stupito e offeso. La mia vita non è stata se non un gioco di rischi. L'ho esposta mille volte ... Com'è dunque possibile, a proposito di me, parlare di "vita preziosa", del "dovere di non espormi", e di simili luoghi comuni? Io non sono un letterato dello stampo antico, in papalina e pantofole. È forse più facile custodire il vento che me. [....] Io sono un soldato, ho voluto essere soldato, non per stare al caffè o a mensa, ma per fare quello che fanno i soldati. Voi volete salvare la mia vita preziosa, voi mi stimate oggetto di museo, da custodire nella stoppa e nella tela da sacchi. Ebbene, ecco, io getto la mia vita, soltanto pel piacere di contraddirvi e di gettarla. (Sodini 1931: 433-434)

Notevole, quindi, nel proseguio della lettera, la completa indifferenza dimostrata da d'Annunzio per la salvaguardia del ruolo di propagandista evidentemente assegnatogli: per contrastare le «ciarle grossolane» raccolte da «qualche giornale» sarà sufficiente impiegare i poteri straordinari della censura in tempi di emergenza, allontanando così il pur legittimo sospetto che il poeta abbia voluto intervenire nel conflitto unicamente per amplificare la propria fama:

Basta dare ordine severissimo che sia soppresso qualunque accenno che mi riguardi, di qualsiasi natura, per tutta la durata della guerra ... Faccia che non sia fiaccata la mia forza, che non sia stroncata la mia fede. Non consideri il pregiudizio vano ma la realtà viva. Abbia cura della mia anima che non è ignobile. (Sodini 1931: 434-435)

In effetti Salandra finì per acconsentire e così d'Annunzio poté rischiare la pelle a suo piacimento: il 7 agosto era già in volo su Trieste con Giuseppe Miraglia, lanciando manifestini sulla città. Non si comprenderà mai abbastanza bene lo spirito giocoso e persino fanciullesco con cui d'Annunzio affronta sconsideratamente il pericolo: come nota ancora Sodini, fra le impressioni del volo triestino, durato tre ore, d'Annunzio è capace di annotare nel suo taccuino i versi della Vispa Teresa: 
«Vivendo, volando, che male ti fo?» ${ }^{6}$ (Sodini 1931: 435); molti anni dopo, nel 1963, l'episodio verrà ricordato da Luciano Bianciardi nell'articolo Un volo una canzone: questa la sua guerra ${ }^{7}$. Ma a qualcuno, vivendo e volando, d'Annunzio fa davvero male: almeno così pare a leggere l'umoroso commento di Gaetano Salvemini in una lettera del 1 ottobre 1915, da San Sepolcro: «d'Annunzio lo mandano sui velivoli a seminar proclami: io preferirei buttarmi giù dal velivolo, anzi che fare il buffone: voglio fare il soldato sul serio e lo sto facendo sul serio» (Salvemini 1984: 203-204).

Non pare cosa da revocare in dubbio, peraltro, che anche d'Annunzio abbia fatto il soldato sul serio, se si bada con occhio il più possibile obiettivo, in rapida sintesi e senza mettere nel computo promozioni e medaglie, alle vicende che seguiranno: il 15 gennaio 1916, d'Annunzio in compagnia del pilota Luigi Bologna ammara bruscamente su una barena della laguna veneta, rimanendo ferito alla tempia e al sopracciglio destro. Il giorno dopo è di nuovo in volo su Trieste e solo dopo più di un mese si accorge che la capacità visiva dell'occhio destro è notevolmente diminuita. Consultatosi con i medici, gli viene prescritto un periodo di assoluto riposo, al buio, che non servirà a salvargli la vista dell'occhio offeso. Nella convalescenza, che terminerà solo il 15 maggio, d'Annunzio scrive com'è noto il Notturno. Il 13 settembre lo ritroviamo in volo su Parenzo, sempre con Luigi Bologna, in azioni di bombardamento; fra il 9 e il 12 ottobre, fattosi assegnare alla $45^{\text {a }}$ divisione di fanteria partecipa alla conquista di quota 363 sul Veliki. Il 1917 è l'anno in cui, il 27 gennaio, muore la madre del Poeta; a maggio torna al volo con diversi bombardamenti sul Carso (in questo stesso mese invia al generale Cadorna un'accurata relazione sull'impiego bellico dell'aviazione); il 3 agosto partecipa all'attacco su Pola, ripetuto poi nella notte fra l'8 e il 9; il 21 agosto il suo aereo viene colpito più volte e d'Annunzio rimane leggermente ferito al polso; il 4 ottobre partecipa al bombardamento di Cattaro; dopo la disastrosa rotta di Caporetto tenta di rincuorare i soldati con i suoi numerosi discorsi. Nel 1918 partecipa, il 10 e l'11 febbraio, alla cosiddetta "Beffa di Buccari", il 15 giugno bombarda gli austriaci nell'offensiva del Montello, il 17 luglio vola di nuovo su Pola, il 9 agosto partecipa alla notissima impresa di Vienna lanciando migliaia di manifestini che invitavano gli austriaci alla resa; dal 24 ottobre al 2 novembre è ancora in volo a supporto dell'offensiva finale che porterà all'armistizio del giorno successivo. In tutto questo periodo d'Annunzio alterna le attività militari alle numerose orazioni, non rinunciando peraltro a intervalli di puro relax e annotando sui Taccuini tutto quel che ritiene notevole, e saranno ad esempio i dettagli tecnici di un volo, gli occhi e la dignità di un prigioniero rumeno, oppure la fucilazione di alcuni ammutinati o la descrizione di un pranzo o dell'aspetto fisico e dell'abbigliamento dei soldati. I

${ }^{6}$ In realtà l'annotazione sul taccuino LXXIX recita precisamente: «Vivendo, volando / che male gli fo?» (d'Annunzio 1965: 741).

${ }^{7}$ Il testo dell'articolo (pubblicato su Il Giorno del 15 marzo 1963) si legge ora in Bianciardi (2002: 31-40). 
Taccuini dannunziani rappresentano indubbiamente una delle testimonianze più straordinarie dell'evento bellico e tuttavia una sistematica ricomposizione tratta dalle proprie opere e assemblata in un nuovo contesto autobiografico si trova nel progetto editoriale de Il fastello della mirra risalente al 1926 ma andato in porto molto tempo dopo, ovvero quasi ai giorni nostri, per la cura di Angelo Piero Cappello (d'Annunzio 2004). Va inoltre considerata, in aggiunta alla già menzionata attività oratoria, la copiosa produzione poetica ora esclusivamente indirizzata ai fini bellici, di cui Giorgio Bàrberi Squarotti ha fornito un esemplare resoconto in un convegno tenutosi al Vittoriale nel 1994 (Bàrberi Squarotti 1996).

Alla guerra di d'Annunzio guarda naturalmente con occhi ora ammirati ora disillusi l'intera comunità culturale italiana: innumerevoli sono i commenti, moltissimi gli spunti creativi che il personaggio bellico offre agli scrittori nostrani, tanto che in questa sede, per dare almeno un'idea della variegata popolarità del Pescarese, converrà riferire solo di alcuni esempi. Così, se nel Trono dei poveri di Marino Moretti d'Annunzio è colto senza particolare acredine nella sua parabola da poeta sentimentale a guerriero (Zollino 2014: 103-107), Palazzeschi si scaglia apertamente contro il Poeta nei suoi Due imperi ... mancati:

Gabriele d'Annunzio apre e chiude la malaugurata stagione della guerra. La guerra d'Italia come fu fatta altro non e che una spacconata d'Annunziana senza senso e senza profitto.

E [sic] ve l'ha guarnita per tutti i suoi giorni, infiorata, incoccardata, di inni, odi e canzoni, orazioni, invocazioni, imprecazioni, inaugurazioni commemorazioni e avventure d'ogni specie; sulla terra e per l'aria sotto e sopra l'acqua, come se si fosse trattato di una grande partita ginnastica, un torneo nel quale tutta la gioia dei muscoli e dei polmoni dovessero avere a pieno il loro sfogo.

Senza neppure domandarsi che razza di guerra fosse mai quella che si doveva ancora combattere, senza curarsi come fossero gli uomini ai quali veniva imposta, nulla. Colla testa alta, e avanti! Vedono ancora, tali uomini, i popoli come le plebi di migliaia di anni fa, e vivono nell'ebbrezza di risuscitare Leonidi, e guerre puniche, centauri, aquile romane, ali di vittorie, rottami di grandezze estinte, come se i secoli, se i millenni non fossero passati su questa umanità.

[...] Raspategli bene addosso, e sapete che cosa ci troverete, in fondo? Un ufficiale di cavalleria.

Creature viventi fuori della realtà e della vita, creatori del vuoto. [...] Inconsciamente, divengono pericolosi strumenti che abili mani calcolatrici sanno bene sfruttare e manovrare. Oh! Quelle conoscono che sia realtà, e vivono al di sotto delle nuvole, e se ne servono. (Palazzeschi 1920: 192-194)

L'analisi particolarmente lucida di Palazzeschi non può tuttavia esimersi dal constatare il largo consenso che ha accompagnato e favorito l'intraprendenza dannunziana: «Avrei mai scritto queste parole, contro un poeta, se non mi ci aveste trascinato per la gola? se non mi aveste voi imposto il dovere sacrosanto di segnalarlo come un frutto avvelenato?» (Palazzeschi 1920: 198). Non per questo, però, Palazzeschi si mostra disponibile a giustificare la vanità dannunziana: 
Dopo avervi tutti mobilitato durante la sua vita acciò lo ritenessi [sic] grande abbastanza, più grande d'ogni soprannaturale grandezza, perché la vita sua fosse bella, bella, bella! Gabriele d'Annunzio ora è vecchio, la vita bella è passata, e sarebbe passata anche se fosse stata brutta, ora e solo preoccupato dalla morte. Deve egli fare una morte come fu la vita, straordinariamente grande e bella. È pronto per essa a mettere a disposizione quaranta milioni di persone perché glie la facciano fare come vuole lui, senza guardare alla spesa. Di tutte ne ha tentate per terra nell'acqua per l'aria, si vede proprio che di là non ce l'hanno voluto, o che nessuna di quelle sperimentate gli sembrò degna di lui. Vuole finire sì alatamente da essere annoverato sui calendari nelle colonne dei Martiri! Martire! Semplice e sublime parola. Dare la vita! Questa cosa sì cara sì grande per chi seppe comprenderla! La vita! Ma, un momento, caro signore, voi ci date la vita, sta bene, ma vediamo un po' di che si tratta, anche noi dobbiamo pur sapere che razza di mercanzia prendiamo, se aveste avuto trent'anni... o quaranta al massimo... certo il dono sarebbe stato grande, e lo avremmo accettato con entusiasmo, ma ne avrete fra un pochino... sessanta, se non erro. Ehi! È un po' tardi eh? ce la date in condizioni un po' avariate, quando tutto il buono è esaurito, e la polpa ve la siete pappata tutta per voi, e avete succhiato e rosicchiato fino in fondo, ma questa caro signore non è altro che la lisca della vostra vita, e noi cosa siamo diventati, il gatto? (Palazzeschi 1920: 199-200)

Anche Vitaliano Brancati, in un dialogo delle Avventure di Tobaico (1946) porrà in termini problematici l'ossessione di d'Annunzio per la guerra, che così si riverbera sul superficiale protagonista:

«Il famoso Giovanni Verga?» disse Tobaico. «Io ho letto I Malavoglia, ma non mi sono piaciuti, perché francamente non fa piacere incontrare in ogni pagina, due e tre volte, il poverino, la poveretta, il poveraccio. Del resto anche Manzoni scrive così, e non mi piace. I poeti devono abbellire la realtà, e non farla vedere più brutta di com'è ... Mi piace d'Annunzio!»

«A me d'Annunzio non piace» disse Guzzardi, «perché ha sempre la testa nella guerra!» «La guerra» disse Tobaico, «io non la farei! Ma i popoli devono farla per essere grandi e potenti!»

«Per forza i popoli devono essere potenti?»

«Certo, se vogliono essere anche ricchi, ben vestiti, e abitare in casa comode!»

«Ma la guerra manda molte persone ad abitare nei cimiteri!»

«Io, le ripeto, la guerra non la farei ...». (Brancati 1982: 117) ${ }^{8}$

Fra i commentatori delle imprese belliche dannunziane c'è poi, assai tempestivamente, lo stesso Poeta che con la pubblicazione de La beffa di Buccari (1918) aggiunge un'altra tessera letteraria a complemento del proprio personaggio: la discreta mistificazione dell'azione di guerra avalla un falso storico senza tuttavia perpetrarlo fino in fondo ${ }^{9}$, dal momento che la nave nemica, che peraltro era un

${ }^{8}$ Per il rapporto fra Brancati e d'Annunzio si veda ora l'interessante indagine di Luca Danti, «Brancati e d'Annunzio: i piaceri del Piacere», in corso di pubblicazione sulla Nuova rivista di letteratura italiana.

${ }^{9}$ Nessuna nave militare, infatti, fu colpita nell'azione (Ledda 1996: 73-86). 
modesto piroscafo, viene descritta nel suo «inclinarsi» senza nulla dire del presunto affondamento, suggerito tuttavia dal testo:

Scorgiamo la massa scura inclinarsi tra qualche battito di bagliori come d'occhi che tentino di aprirsi e si richiudano per morire. Un vocìo confuso, un gridìo sparso, un accendersi e un agitarsi di fanali, colpi di fuoco rari, qua e là: l'allarme! (d'Annunzio 1918: 45)

La ricostruzione, evidentemente di parte, lascia tuttavia intatta la sostanza delle cose per quanto riguarda il coraggio fisico del Poeta e dei suoi trenta compagni d'azione, che certamente hanno rischiato perlomeno una clamorosa cattura da parte degli austriaci nel compiere l'impresa: da ricordare peraltro che «funzionari della propaganda viennese» avevano già da tempo provveduto ad impiccare in effigie d'Annunzio, come documentato anche da reperti fotografici dell'epoca (Roncoroni / Rossi 1986: 36). E tuttavia non piacerà a Federigo Tozzi questa Beffa di Buccari, che offre lo spunto per un consuntivo sul Poeta:

Sembrava che la guerra potesse dargli l'occasione di rendere il suo parossismo pànico; e invece la guerra nell'arte d'annunziana ha minore importanza delle cose insignificanti che continuano, com'è naturale, a far parte dell'apparato estetico. Egli è restato qual era. E si capisce subito quando si ricorda il terzo libro delle laudi, che è il più bello e il più equilibrato: dove si trova il miracolo di Versilia. Il quale basterebbe da solo a dare modo di analizzare tutto quanto di più alto ha scritto il d'Annunzio. (Tozzi 1993: 189)

Tozzi si pone dunque sulla linea di Serra, per cui la guerra non cambia nulla: e dunque non cambia nemmeno d'Annunzio. Questi in effetti non fa altro, anche in questa occasione, che ripetersi:

Gabriele d'Annunzio è soltanto un modesto ma appassionato imitatore di se stesso. Lo dico senza dimenticarmi che egli è il nostro più grande scrittore, rimpiangendo i bei tempi che la sua arte mi pareva un meriggio sgargiante; dove la mia giovinezza ha trovato liberamente la strada. E altri giovini lo stesso. Ma ora mi rivolto a leggere certe ciance. (Tozzi 1993: 189-90)

L'intempestivo articolo, pubblicato ne Il Tempo del 10 aprile 1918, costerà caro allo scrittore senese, poiché Giovanni Beltrami, direttore editoriale di Treves, irritato per l'articolo rivolto contro l'autore di punta della stessa casa editrice milanese, deciderà di rimandare per il momento la pubblicazione di Con gli occhi chiusi, ricordando a Tozzi, che stava trascorrendo il periodo bellico in un ufficio della Croce Rossa, che "d'Annunzio [...] da quattro anni scrive col rischio di sua vita pagine di poesia immortale»(Cesarini 2002: 209). Ecco allora che Tozzi pubblica un articolo che appare di riparazione (e quindi non del tutto immune da sospetti di opportunistica convenienza) dove però non si trovano i luoghi comuni del dannunzianesimo più vieto, quanto invece l'importante puntualizzazione del 
principio di libertà estetica (già accennato, come abbiamo visto nell'articolo sulla Beffa di Buccari) che d'Annunzio ha saputo introdurre nella letteratura italiana:

d'Annunzio $[\ldots]$ ha soddisfatto con più immediatezza la nostra sensibilità, avendo potuto mettere nei giovani un insegnamento che appunto, perché essenzialmente estetico, ci lascia poi liberi e ci suggerisce il mezzo di cercare a noi stessi una decisiva individualità [...] Senza Gabriele d'Annunzio nessuno di noi sarebbe in grado né di leggere né di scrivere. Ed è assolutamente indispensabile ricordare che qualunque rinnovamento letterario possa dare l'Italia, noi dobbiamo esserne grati soltanto a lui. In questo senso, ripeto, egli è l'unico nostro maestro moderno. (Tozzi 1993: 245) (10 $^{10}$

A guerra terminata, d'Annunzio prolungò e mise a frutto le proprie esperienze militari con la conquista di Fiume, terminata, com'è noto, con il cosiddetto Natale di sangue del 1920: la delusione per l'attacco subito dalle stesse truppe regolari italiane si aggiunse così a quelle patite per il mancato rispetto del Patto di Londra (in cui però non era contemplata Fiume) da parte dei nostri alleati e per la stipula del trattato di Rapallo, che per il momento escludeva Fiume dalle pertinenze territoriali italiane. In conseguenza di ciò d'Annunzio, nell'articolo «A faccia a faccia», pubblicato sulla Gazzetta del Popolo di Torino del 24 novembre 1921 e successivamente, negli Stati Uniti e in traduzione inglese, sul New York American, è capace di guardare retrospettivamente all'esperienza bellica con modi e toni del tutto inusitati:

Quando mai nella storia del mondo un grave evento lasciò dietro di sé una più grande delusione? Fummo tanto ingenui da credere che la guerra da noi combattuta avrebbe rinnovato la vita. Pensammo che la strage preparasse gli spazi mistici per le apparizioni ideali. Pensammo che la terra prendesse il corpo orizzontale dell'uomo come misura unica per misurare il più vasto Destino, e che, saziata di carne, ce la rendesse in spirito. Pensammo che il carnaio dissolvendosi generasse i fermenti sublimi. Pensammo che la libertà dell'anima si levasse là dove si sprofondava il peso mortale. Pensammo che, ove più larga era l'offerta tanto più alto dovesse essere il prodigio.

E troppo presto ci accorgemmo di aver combattuto per mantenere in movimento la vecchia macchina costrittiva della ingiustizia e del servaggio. (ora in Mariano 1978: 54)

Avviandomi ormai alla conclusione di questa rapida indagine, osserverò ancora che se in Italia, per forza di cose, la considerazione delle azioni di guerra dannunziane si sottopone alle modalità del dibattito politico interno, all'estero la fama del poeta guerriero, almeno fra le nazioni che non furono nemiche dell'Italia nel primo conflitto mondiale, appare decisamente meno problematica. Così in un'intera pagina a fumetti del New York Daily Mirror del primo settembre 1934, significativamente intitolata Above the crowd, campeggia un'immagine del volto di d'Annunzio, già monocolo e con il berretto d'ordinanza dell'aviazione, affiancata

10 Così nell'articolo «Giovanni Verga e noi», pubblicato ne Il Messaggero della domenica del 17 novembre 1918. 
da un disegno rappresentante una colonna motorizzata lanciata all'assalto della città di Fiume e dalla seguente didascalia:

\section{SPIRIT OF ITALY}

He will always be remembered as the eroic poet who, forsaking his pen for the sword, performed amazing deeds for his country. His magnificent coup of seizing the Adriatic port of Fiume in 1919 in defiance of Jugoslavia and all the entente powers saved Fiume for Italy.

With only 1.600 men and a few armored cars he took the city! (d'Annunzio 1994, tavola fuori testo fra le pp. 80 e 81 )

La pagina, illustrata dal disegnatore Stookie Allen, prosegue quindi con l'immagine di un aereo italiano caduto affiancato da un veicolo di soccorso; due portantini si allontanano recando un corpo in barella, quello del poeta, come si evince dal commento: "He lost an eye in the war but even that didn't curb his flaming spirit. Time and again he was cited for bravery and given medals». Alla successiva immagine di Vienna sorvolata dai biplani italiani che lanciano volantini è sottoposta invece la didascalia:

Gabriele bombarded Vienna with POETRY!

The poetry was propoganda [sic] and did more good than any real bombs could have.

He was hailed as the embodiment of Italy's undying spirit! (d'Annunzio 1994, tavola fuori testo fra le pp. 80 e 81 )

La pagina del popolare giornale newyorkese, inviata il 12 settembre 1934 da Veniero d'Annunzio al padre ormai irrimediabilmente confinato ai margini della vita nazionale, si conclude, nell'angolo in basso a destra, con il curioso autoritratto del disegnatore Stokie Allen che si produce nel saluto romano, esclamando appunto nel fumetto, in italiano: «Saluta!» (d'Annunzio 1994, tavola fuori testo fra le pp. 80 e 81).

\section{RIFERIMENTI BIBLIOGRAFICI}

AnTOGNINI, Tom (1938): Vita segreta di Gabriele d'Annunzio, Milano, Mondadori. AA. VV. (1996): d'Annunzio e la guerra: Atti del convegno internazionale Gabriele d'Annunzio e la guerra. Gardone Riviera, 17-19 novembre 1994, Milano, Mondadori

BÀRBERI SQUAROTTI, Giorgio (1996): «Le immagini della guerra», in AA.VV., d'Annunzio e la guerra, Milano, Mondadori, pp. 195-217.

BIANCIARDI, Luciano (2002): Un volo e una canzone. d'Annunzio: l'eroe immoralista della piccola Italia, pref. di Giacomo D’Angelo, Milano, ExCogita. 
BRANCATI, Vitaliano (1982): «Avventure di Tobaico», in Vitaliano Brancati, Sogno di un valzer e altri racconti, a cura di Enzo Siciliano, nota ai testi di Rita Verdirame, Milano, Bompiani, pp. 105-152.

CAMPANA, Dino (1985): Souvenir d'un pendu. Carteggio 1910-1931 con documenti inediti e rari, a cura di Gabriel Cacho Millet, Napoli, Edizioni Scientifiche Italiane.

CESARINI, Paolo (2002): Tutti gli anni di Tozzi, a cura di Carlo Fini, cronologia e indici di Luigi Oliveto, Montepulciano, Le Balze.

CozZANI, Ettore (1930): Gabriele d'Annunzio. La preparazione e l'opera di guerra, Milano, L'Eroica.

CoZZANI, Ettore (1963): Come giungemmo alla Sagra dei Mille, Milano, L'Eroica.

D'ANNUNZIO, Gabriele (1918): La beffa di Buccari, con aggiunti La canzone del Quarnaro, Il catalogo dei trenta di Buccari, Il cartello manoscritto e Due carte marine, Milano, Treves.

D'ANNUNZIO, Gabriele (1947): Prose di ricerca, di lotta, di comando, di conquista, di tormento, d'indovinamento, di rinnovamento, di celebrazione, di rivendicazione, di liberazione, di favole, di giochi e di baleni, vol. I, Milano, Mondadori.

D'ANNUNZIO, Gabriele (1965): Taccuini, a cura di Enrica Bianchetti e Roberto Forcella, Milano, Mondadori.

D’ANNUNZIO, Gabriele (1994): Carteggio inedito con il figlio Veniero (1917-1937), (Periodo Usa), a cura di Maria Grazia Di Paolo, Milano, Mursia.

D’ANNUNZIO, Gabriele (2004): Il fastello della mirra, a cura di Angelo Piero Cappello, pref. di Annamaria Andreoli, Firenze, Vallecchi.

GADDA, Carlo Emilio (1974): La meccanica, Milano, Garzanti.

LANZALONE, Giovanni (1919): Speranze umane. [Ristampa del Suicidio della Guerra e degli Epigrammi di Guerra con molte aggiunte e correzioni], Reggio Emilia, Guidetti.

LEDDA, Elena (1996): «Buccari: "per osare 1'inosabile". Storia di una beffa», in AA.VV., d'Annunzio e la guerra, Milano, Mondadori, pp. 73-86.

MARIANO, Emilio (1978): «Il San Francesco di Gabriele d'Annunzio», Quaderni del Vittoriale 12, pp. 9-82.

OJETTI, Ugo (1957): d'Annunzio amico maestro soldato, 1894-1944, Firenze, Sansoni.

OSTENC, Michel (1997): «Gabriele d'Annunzio et l'entrée en guerre de l'Italie dans la presse française de 1915», Guerre mondiales et conflits contemporains 185, pp. 89-110.

PALAZZESCHI, Aldo (1920): Due imperi ... mancati, Firenze, Vallecchi.

PANZINI, Alfredo (1916): La Madonna di Mamà. Romanzo del tempo della guerra, Milano, Treves.

PANZINI, Alfredo (1923): Diario sentimentale: dal luglio 1914 al maggio 1915, Milano, Mondadori.

Roncoroni, Federico / Rossi, Bruno (a cura di) (1986): d'Annunzio e il Corriere, Milano, Editoriale del Corriere della Sera. 
Salvemini, Gaetano (1984): Carteggio 1914-1920, a cura di Enzo Tagliacozzo, Bari, Laterza.

SCALIA, Gianni (a cura di) (1973): La cultura italiana del '900 attraverso le riviste. Volume IV: Lacerba La Voce (1914-1926), Torino, Einaudi, pp. 555-563.

SERRA, Renato (1973): Esame di coscienza di un letterato e ultime lettere dal campo, intr. di Carlo Bo, Roma, Newton Compton.

TozzI, Federigo (1993): «La beffa di Buccari», in Federigo Tozzi, Pagine critiche, a cura di Giancarlo Bertoncini, Pisa, ETS, pp. 189-190.

Zollino, Antonio (2014): La bella sorte. Il personaggio d'Annunzio nella letteratura e nella vita culturale italiana, Lugano, Agorà \& co. 